\title{
Calculation of the particles transfer in a porous medium
}

\author{
Olga Shirokova ${ }^{1, *}$ \\ ${ }^{1}$ Moscow state university of civil engineering, Yaroslavskoye shosse, 26, Moscow, Russia, 129337
}

\begin{abstract}
The transfer and deposition of solid particles in porous rocks are encountered in many fields of science and technology, including the design and construction of waterproof foundations and hydraulic structures. The paper considers polydisperse suspesion with suspended particles of various types moving in the porous medium at the same velocity. The asymptotics of the solution of the filtration problem with various linear filtration coefficients is constructed. A numerical calculation of the asymptotics is performed for the case of two different types of particles.
\end{abstract}

\section{Introduction}

The transfer and retention of solid suspended particles of suspension and colloids in the porous medium is one of the main problems of underground fluid mechanics [1]. Such problems are encountered in many fields of science and technology, in many fields of science and technology, including the design and construction of waterproof structures and hydraulic structures.

Classical filtration models of a suspension in the porous medium consider a flow of a carrier fluid with the identical suspended particles [2, 3]. Porous medium has a complex structure, and various factors can influence the retention of particles in the filter pores: electrical forces, gravity, dispersion, viscosity, etc. If the particle and pore sizes are close, then the determining mechanism of pore capture is size-exclusion. The mathematical model of deep bed filtration includes the equation of mass balance and the kinetic equation of deposit growth rate, forming a quasilinear hyperbolic system. The kinetic equation determines the growth rate of the deposit in proportion to the concentration of suspended particles. The coefficient of proportionality is called the filtration coefficient. In the simplest cases, the filtration coefficient is assumed to be constant. A linear relationship describing the change in the growth rate deposit with increasing the concentration deposit is used in more complex models. Analytic and numerical solutions of the classical filtration problem are known [3-9]. Models with a complicated equation of mass balance take into account the change in porosity and permeability porous medium when forming a deposit [10-14].

Consider a suspension with suspended particles of two types. Particles, differing in size or shape, move in the porous medium at the same velocity. It is assumed that particles of

* Corresponding author: shirokovaol@mgsu.ru 
different types interact differently with pores and have different filtration coefficients. In the paper the asymptotic solution of the filtration problem is constructed near the concentrations front of suspended and retained particles and a numerical calculation of the asymptotics is performed.

\section{Formulation of the problem}

In the domain

$$
\Omega=\{0<x<1, t>0\}
$$

a mathematical model is given by a system of equations

$$
\begin{gathered}
\frac{\partial C_{i}}{\partial t}+\frac{\partial C_{i}}{\partial x}+\frac{\partial S_{i}}{\partial t}=0 \\
\frac{\partial S_{i}}{\partial t}=\Lambda_{i}(\mathbf{S}) C_{i} ; i=1, \ldots, n
\end{gathered}
$$

with the boundary condition

$$
x=0: C_{i}=p_{i} .
$$

and the initial conditions

$$
t=0: C_{i}=0, S_{i}=0 .
$$

Here $\mathbf{S}=\left(S_{1}, \ldots, S_{n}\right)$; the filtration coefficients $\Lambda_{i}(\mathbf{S})$ are continuous and positive; the constants $p_{i}>0$.

At the initial moment the porous medium does not contain any particles. A suspension with a constant concentration $p_{i}$ of suspended particles begins to flow into the filter inlet. The concentrations front of the suspended and retained particles moves from the filter inlet $x=0$ to the outlet $x=1$ at a constant velocity $v=1$ and divides the domain $\Omega$ into two subdomains $\Omega_{0}=\{0<x<1,0<t<x\}$ and $\Omega_{1}=\{0<x<1, t>x\}$. In $\Omega_{0}$ the system (1)(4) has a zero solution; in $\Omega_{1}$ the solution is positive. Since conditions (3) and (4) are not matched at the origin, the solution $C_{i}(x, t)$ has a strong discontinuity at the concentrations front, the characteristic straight line $t=x$.

The solution $S_{i}(x, t)$ is continuous in $\Omega$ and has a weak discontinuity on the concentrations front.

We define a condition on the characteristic

$$
\left.S_{i}\right|_{t=x}=0 .
$$

In the domain $\Omega_{1}$ the problem (1) - (4) is equivalent to the Goursat problem (1) - (3), (5).

In the characteristic variables (Riemann variables) $\tau=t-x, x=x$ in the domain

$$
\tilde{\Omega}_{1}=\{0<x<1, \tau>0\}
$$


the system $(1)-(3),(5)$ takes the form

$$
\begin{aligned}
& \frac{\partial C_{i}}{\partial x}+\frac{\partial S_{i}}{\partial \tau}=0 ; \\
& \frac{\partial S_{i}}{\partial \tau}=\Lambda_{i}(\mathbf{S}) C_{i}
\end{aligned}
$$

with initial and boundary conditions

$$
\begin{array}{r}
x=0: C_{i}=p_{i} ; \\
\tau=0: S_{i}=0 ; i=1, \ldots, n .
\end{array}
$$

\section{The exact solution on the concentrations front}

Let us determine the concentrations of suspended particles on the concentrations front. Substituting (7) into (6)

$$
\frac{\partial C_{i}}{\partial x}+\Lambda_{i}(\mathbf{S}) C_{i}=0 ; i=1, \ldots, n
$$

The concentrations front is given by formula $\tau=0$. According to condition (5), on the concentrations front equation (10) has the form

$$
\frac{\partial C_{i}}{\partial x}+\Lambda_{i}(0) C_{i}=0,\left.C_{i}\right|_{x=0}=p_{i}
$$

The solution of (11)

$$
C_{i}=p_{i} e^{-\Lambda_{i}(0) x}, i=1, \ldots, n
$$

The formula (12) gives the exact solution on the concentrations front.

\section{Asymptotics near the concentrations front}

Assume that the filtration coefficients linearly depend on the concentrations of $n$ types of retained particles

$$
\Lambda_{i}(\mathbf{S})=\lambda_{i}+\sum_{k=1}^{n} a_{k} S_{k}, i=1, \ldots, n
$$

where $a_{k}$ are constants.

In the neighborhood of the concentrations front $\tau=0$ the solution of problem (6) - (9) can be obtained in the form

$$
S_{i}=S_{i}^{1} \tau+\frac{1}{2} S_{i}^{2} \tau^{2}+O\left(\tau^{3}\right) ; \quad C_{i}=C_{i}^{0}+C_{i}^{1} \tau+O\left(\tau^{2}\right) ; i=1, \ldots, n
$$


Here $C_{i}^{0}$ are given by the formulas (12), the expansions coefficients $S_{i}^{1}, S_{i}^{2}, C_{i}^{1}$ depend on the variable $x$.

Substituting the expansions (14) into equations (6) and (7) and equating the terms of the same powers of $\tau$, we obtain a system of recurrent algebraic and differential equations for the leading terms of the asymptotics

$$
\begin{gathered}
S_{i}^{1}=\lambda_{i} C_{i}^{0} \\
\left(C_{i}^{1}\right)^{\prime}+\lambda_{i} C_{i}^{1}+C_{i}^{0} \sum_{k=1}^{n} a_{k} S_{k}^{1}=0 ; \\
S_{i}^{2}=\lambda_{i} C_{i}^{1}+C_{i}^{0} \sum_{k=1}^{n} a_{k} S_{k}^{1} ; i=1, \ldots, n .
\end{gathered}
$$

The initial condition for the differential equation (16) is obtained from (3)

$$
C_{i}^{1}=0 ; \quad i=1, \ldots, n \text {. }
$$

The solution of the system (15) - (17)

$$
\begin{gathered}
S_{i}^{1}=\lambda_{i} p_{i} e^{-\lambda_{i} x} \\
C_{i}^{1}=p_{i} e^{-\lambda_{i} x} \sum_{k=1}^{n} p_{k} a_{k}\left(e^{-\lambda_{k} x}-1\right) ; \\
S_{i}^{2}=p_{i} e^{-\lambda_{i} x} \sum_{k=1}^{n} p_{k} a_{k}\left(\left(\lambda_{i}+\lambda_{k}\right) e^{-\lambda_{k} x}-\lambda_{i}\right) .
\end{gathered}
$$

Substituting the terms (12), (19) - (21) into the expansions (14) and performing an inverse change of variables, we obtain the asymptotics of problem (1) - (4) in the domain $\Omega_{1}$ near the concentrations front

$$
\begin{gathered}
S_{i}(x, t)=\lambda_{i} p_{i} e^{-\lambda_{i} x}(t-x)+\frac{1}{2} p_{i} e^{-\lambda_{i} x} \sum_{k=1}^{n} p_{k} a_{k}\left(\left(\lambda_{i}+\lambda_{k}\right) e^{-\lambda_{k} x}-\lambda_{i}\right)(t-x)^{2}+O(t-x)^{3} \\
C_{i}=p_{i} e^{-\lambda_{i} x}+p_{i} e^{-\lambda_{i} x} \sum_{k=1}^{n} p_{k} a_{k}\left(e^{-\lambda_{k} x}-1\right)(t-x)+O(t-x)^{2} ; i=1, \ldots, n
\end{gathered}
$$

\section{Numerical simulation}

The numerical calculation of the asymptotic solution is performed for 2-size particles $(n=2)$ for the linear filtration coefficients

$$
\Lambda_{1}=1-S_{1}-S_{2} ; \quad \Lambda_{2}=2-S_{1}-S_{2}
$$

and the initial suspended particles concentrations $p_{1}=1, p_{2}=1$.

Fig. 1 shows 3-D graphs of the suspended and retained particles concentrations separately for each type of particles. 

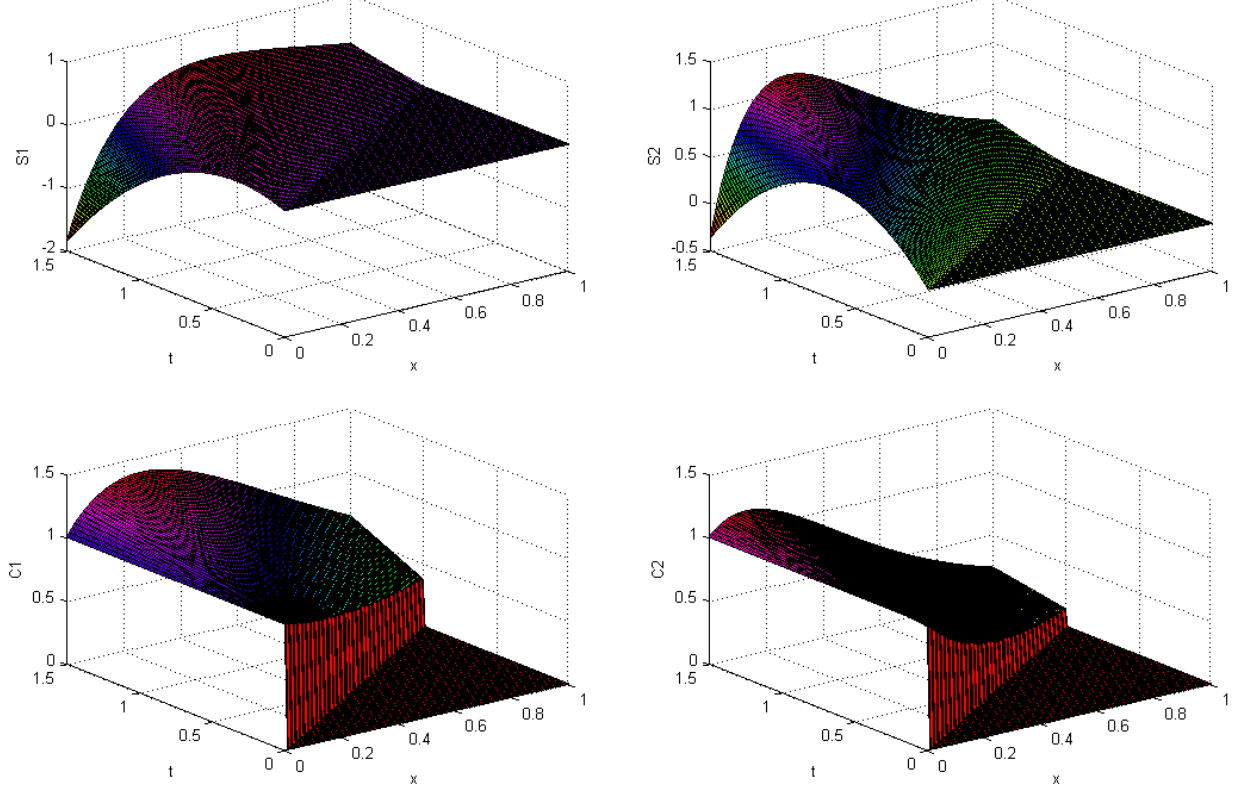

Fig. 1. Suspended and retained particles concentrations $S_{1}(x, t) ; S_{2}(x, t) ; C_{1}(x, t) ; C_{2}(x, t)$.

The graphs of suspended and retained particles concentrations for different fixed $x$ are presented in Fig. 2.
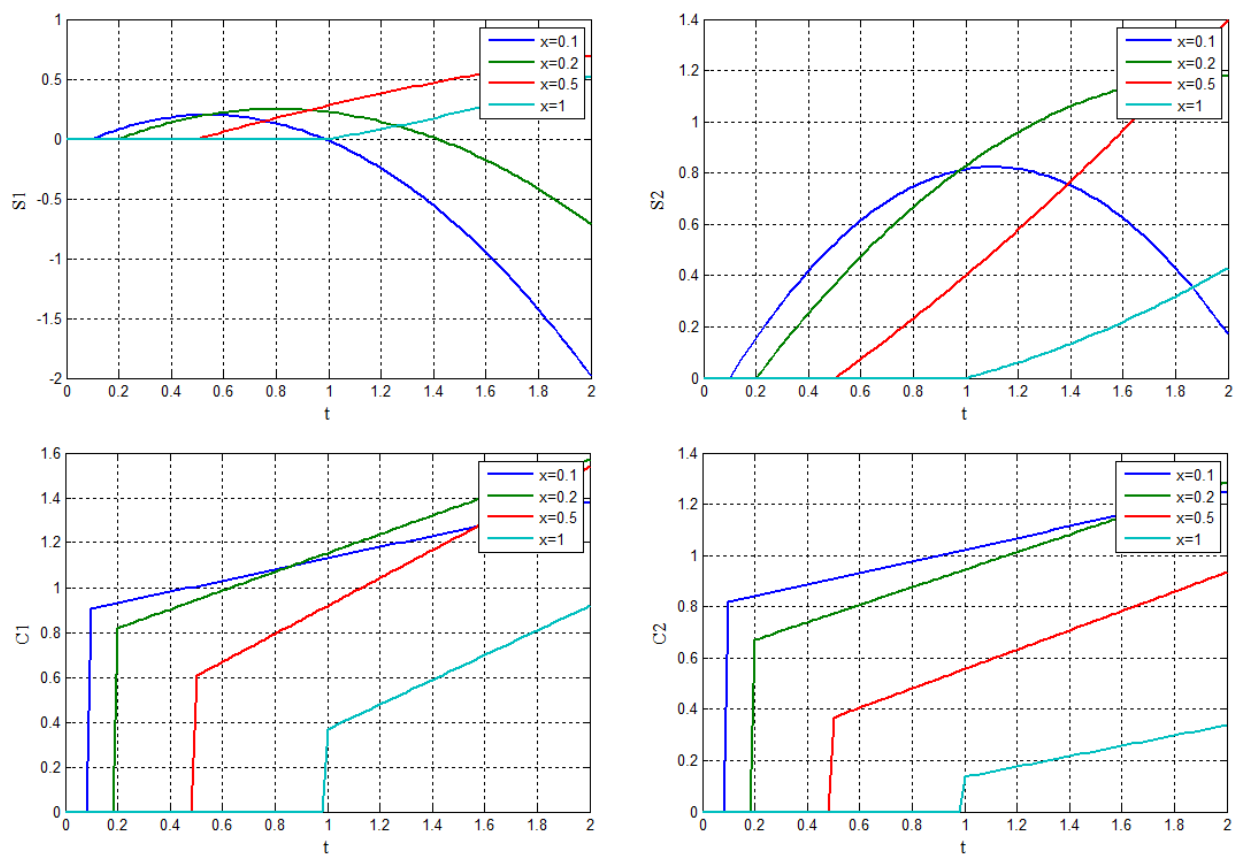

Fig. 2. Particles concentrations $\left.S_{1}(x, t)\right|_{x=c o n s t} ;\left.S_{2}(x, t)\right|_{x=c o n s t} ;\left.C_{1}(x, t)\right|_{x=c o n s t} ;\left.C_{2}(x, t)\right|_{x=c o n s t}$. 


\section{Conclusion}

The asymptotic solution of the problem of particles transfer is constructed near the concentrations front. Particles of $n$ different types move in a porous medium with the same velocity. It is assumed that the filtration coefficients of all types of particles are linearly dependent on the retained particles concentrations.

A numerical calculation of the asymptotic solution for the particles of two different types is carried out. The calculation indicates that the applicability of the asymptotics is limited by the small parameter $\tau=t-x$. Figures $1-2$ show that the asymptotic solution is valid for $\tau<0.4$. For small values of $\tau$, the asymptotics determines the analytic dependence of the solution on the parameters, reducing the amount of laboratory and field research [15].

With increasing $\tau$, the retained particles concentration of the first type begins to decrease and at $\tau \sim 1$ becomes negative. Respectively the suspended particles concentration of the first type exceeds the initial concentration at the filter inlet, which contradicts the physical meaning of the problem.

A further development of the method involves the construction of a global asymptotic solution of the filtration problem on a small limiting deposit.

\section{References}

1 G.I. Barenblatt, V.M. Entov, V.M. Ryzhik, Theory of fluid flows through natural rocks, (Kluwer Acad. Publ. 1989)

2 M. Sharma, Y. Yortsos, AIChE J. 33 (1987) 1636 - 1643

3 J. P. Herzig, D. M. Leclerc, P. Legoff, Ind. Eng. Chem. 62 (1970) $9-35$

4 E.A. Vyazmina, P.G. Bedrikovetskii, A.D. Polyanin, Theor. Found. Chem. Eng. 41, 5 (2007) $556-564$

5 A.D. Polyanin, V.F. Zaitsev, Handbook of nonlinear partial differential equations, (CRC pr. 2003)

6 A. Vaz, D. Maffra, T. Carageorgos, P. Bedrikovetsky, J. Nat. Gas Sci. Eng. 34 (2016) $1422-1433$

7 L.I. Kuzmina, Yu.V. Osipov, Int. J. Comp. Civil Struct. Eng.10 (3) (2014) 17 - 22

8 L.I. Kuzmina, Yu.V. Osipov, Vestnik MGSU 2 (2016) $49-61$

9 Yu.P. Galaguz, G.L. Safina, Procedia Eng. 153 (2016) 157 - 161

10 Z. You, P. Bedrikovetsky, L. Kuzmina, Abstr. Appl. Anal. 2013, ID 680693 (2013)

11 Z. You, Y. Osipov, P. Bedrikovetsky, L. Kuzmina, Chem. Eng. J. 258 (2014) 374-385

12 L.I. Kuzmina, Yu.V. Osipov, Procedia Eng. 111 (2015) 491 - 494

13 L.I. Kuzmina, Yu.V. Osipov, Matec Web Conf. 86, 01005 (2016)

14 Y. Galaguz, G. Safina, Matec Web Conf. 86, 03003 (2016)

15 P.G. Bedrikovetsky, D. Marchesin, F. Checaira, A.L. Serra, E. Resende, J. Petrol. Sci. Eng. 32, 3 (2001) $167-177$ 